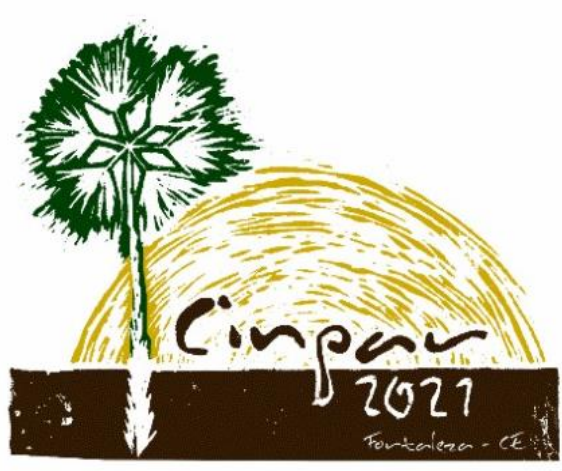

XVII Congresso Internacional sobre Patologia e

Reabilitação das Construções

XVII Congreso Internacional sobre Patología y Rehabilitación de las Construcciones

XVII International Conference on Pathology and Constructions Rehabilitation

FORTALEZA (Brasil), 3 a 5 de junho de 2021

https://doi.org/10.4322/CINPAR.2021.112

\title{
Metodologia GDE/UNB aplicada na avaliação de uma edificação residencial
}

\section{GDE / UNB methodology applied to the evaluation of a residential building}

\author{
Mikhael Ferreira da Silva SANTOS ${ }^{1}$, Cláudio Vidrih FERREIRA ${ }^{2}$, Kelly Cristina Silva Santos ${ }^{3}$ \\ ${ }^{1}$ UFPE, Recife, Brasil, mikhael@ferreiraeng.com \\ 2 Instituto Vidrih, Bauru/SP, Brasil, vidrih@vidrih.com.br \\ ${ }^{3}$ UNIFACEMA, Caxias, Brasil, kellycsantos95@gmail.com
}

Resumo: A metodologia GDE/UnB, também conhecida como PECC/UnB é utilizada para determinar o grau de deterioração de um elemento ou de uma estrutura. Essa metodologia foi desenvolvida por Castro (1994), sendo inicialmente formulada para avaliar obras de arte. Não obstante, percebeu-se a aplicação geral que essa metodologia pode trazer, inclusive para os profissionais ligados de alguma forma à área de manifestações patológicas. Nesse sentido, a metodologia foi adaptada para diversas edificações distintas conforme pode ser encontrada em Lopes (1998), Boldo (2002), Fonseca (2007) e Souza (2009). O princípio da aplicação é a quantificação do grau de deterioração da estrutura em estudo, em outras palavras, é a quantificação do estado atual do elemento estrutural relacionado ao dano do mesmo. O município de Caxias, estado do Maranhão, estando a $360 \mathrm{~km}$ da capital São Luís, está em constante desenvolvimento como pode ser verificado nos últimos anos, pois houve crescimento exponencial do número de edificações. Aliado ao desenvolvimento econômico, diversas edificações apresentam um desempenho abaixo do mínimo aceitável. Uma onda de processos judiciais em construtoras e profissionais da área de construção civil assolam o município, principalmente devido a erros de execução e planejamento. Nesse sentido, o presente trabalho objetiva aplicar a metodologia GDE/UnB na avaliação do grau de deteioração da estrutura de uma edificação residencial situada no município de Caxias/MA. Com base nos resultados, esperam que os profissionais avaliam de maneira quantititativa as manifestações patológicas em edificações residenciais da cidade.

Palavras-chave: GDE/UnB; PECC/UnB; manifestações patológicas; grau de deterioração em estruturas.

\begin{abstract}
The GDE / UnB methodology, also known as PECC / UnB, is used to determine the degree of deterioration of an element or structure. This methodology was developed by Castro (1994), being initially formulated to evaluate works of art. Nevertheless, it was realized the general application that this methodology can bring, even for professionals connected in some way to the area of pathological manifestations. In this sense, the methodology was adapted for several different buildings as can be found in Lopes (1998), Boldo (2002), Fonseca (2007) and Souza (2009). The application principle is the quantification of the degree of deterioration of the structure under study, in other words, it is the quantification of the current state of the structural element related to its damage. The municipality of Caxias, state of Maranhão, being $360 \mathrm{~km}$ from the capital São Luís, is in constant development as can be seen in recent years, as there has been an exponential growth in the number of buildings. Allied to economic development, several buildings perform below the acceptable minimum. A wave of lawsuits in construction companies and professionals in the civil construction area are plaguing the municipality, mainly due to errors in execution and planning. In this sense, the present work aims to apply the GDE / UnB methodology in the evaluation of the degree of deterioration of the structure of a residential building located in the city of Caxias / MA. Based
\end{abstract}


on the results, they expect professionals to quantitatively assess pathological manifestations in residential buildings in the city

Keywords: GDE / UnB; PECC / UnB; pathological manifestations; degree of deterioration in structures.

\section{Introdução}

As manifestações patológicas em edificações está cada vez mais comum. Mão de obra desqualificada, emprego de materiais inadequados, erros de projeto, execução e utilização, entre outras diversas causas, estão impulsionando o aparecimento de uma série de anomalias.

Souza e Ripper (1998) afirmam que os problemas nas estruturas de concreto armado decorrem, em grande parte, de um projeto inadequado e de uma execução descuidada. A gama de profissionais desqualificados que chegam no mercado de trabalho está cada vez mais presente.

A figura 01, trazida por Martins (2003) ilustra a origem percentual de falhas nas edificações em países tais como Bélgica, Grã-Bretanha, Alemanha, Dinamarca e Romênia.

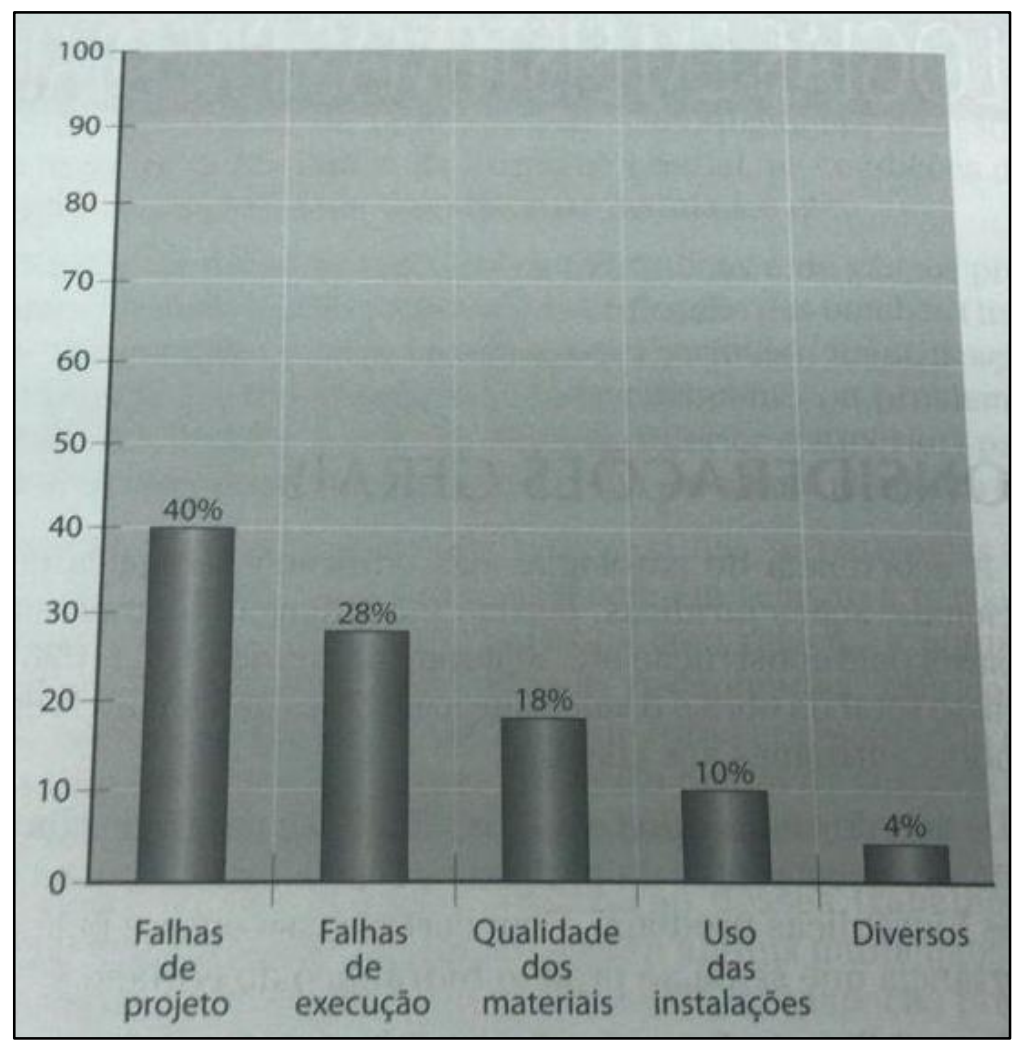

Figura 01. Origem percentual de falhas em edificações. Fonte: Martins (2011).

De fato, a maioria das manifestações patológicas poderiam ser evitados se houvesse projetos bem detalhados, escolha correta de materiais, correta execução, assim como uma maior preocupação com a etapa de prevenção e manutenção. Muitos cuidados são deixados de lado quando se projeta e também durante o uso da edificação o que reduz significativamente o desempenho e sua vida útil (KLIMPLEL; SANTOS, 2010).

Com o decorrer dos anos adquiriu-se muitos conhecimentos acerca da necessidade de manutenção preventiva das estruturas de concreto. Diversas formas de análise de estruturas, como a metodologia GDE/UnB, desenvolvida por Castro (1994), foram desenvolvidas no intuito de ter parâmetros objetivos de prevenção. Nesse sentido, o presente trabalho objetiva aplicar a metodologia GDE/UnB na avaliação do grau de deterioração da estrutura de uma edificação residencial situada no município de Caxias/MA. Com base 
nos resultados, esperam que os profissionais avaliam de maneira quantitativa as manifestações patológicas em edificações residenciais da cidade.

\section{Metodologia GDE/UnB para quantificação do grau de deterioração de uma estrutura}

A metodologia GDE/UnB foi desenvolvida por Castro (1994) no Programa de Pós Graduação em Estruturas e Construção Civil da Universidade de Brasília (PECC - UnB). A metodologia permite realizar a avaliação quantitativa do grau de deterioração de estruturas de concreto, analisando e quantificando as manifestações dos danos e suas evoluções, assim, visa dar criticidade as peças estruturais de uma edificação, viabilizando a definição de prioridades para realização das manutenções.

A metodologia evolui com os trabalhos de Lopes (1998), Boldo (2002), Fonseca (2007), Euqueres (2011) e Verly (2015), buscando aprimorar e resolver lacunas da primeira publicação em 1994. Lopes (1998) verificou que os resultados provenientes de Castro (1994) poderiam levar a resultados muito desconexos, trazendo uma inovação na fórmula do Grau de Deterioração do Elemento (Gde). Bolso (2002), por sua vez, trouxe melhorias na determinação do Grau de Deterioração de Família (Gdf), semelhante ao anterior. Fonseca (2007) utilizou a metodologia GDE/UnB buscando avaliar o grau de deterioração de estruturas de concreto armado e apresentou adaptações, indicando alterações nas formulações dos cálculos do Grau de deterioração da família (Gdf) e do Grau do Dano (D), além disso, apresentou uma alteração nos tipos mais frequentes de dano. Euqueres (2011) fez adaptação com adequação às estruturas de pontes. Verly (2015) publicou o Manual de Aplicação da Metodologia GDE/UnB a Obras de Arte Especiais.

Nesse sentido de desenvolvimento, a metodologia GDE/UnB pode ser aplicada em edificações com diferentes tipos de estruturas. Além do mais, pode ser utilizada como ferramenta para gerir atividades de manutenção corretiva, permitindo indicar os elementos que possuem falhas com maior gravidade e/ou urgência, facilitando tomada de decisão, no âmbito técnico-financeiro das intervenções.

O fluxograma expresso na figura 02 ilustra o esquema de utilização da metodologia GDE/UnB, conforme desenvolvido por Castro (1994).

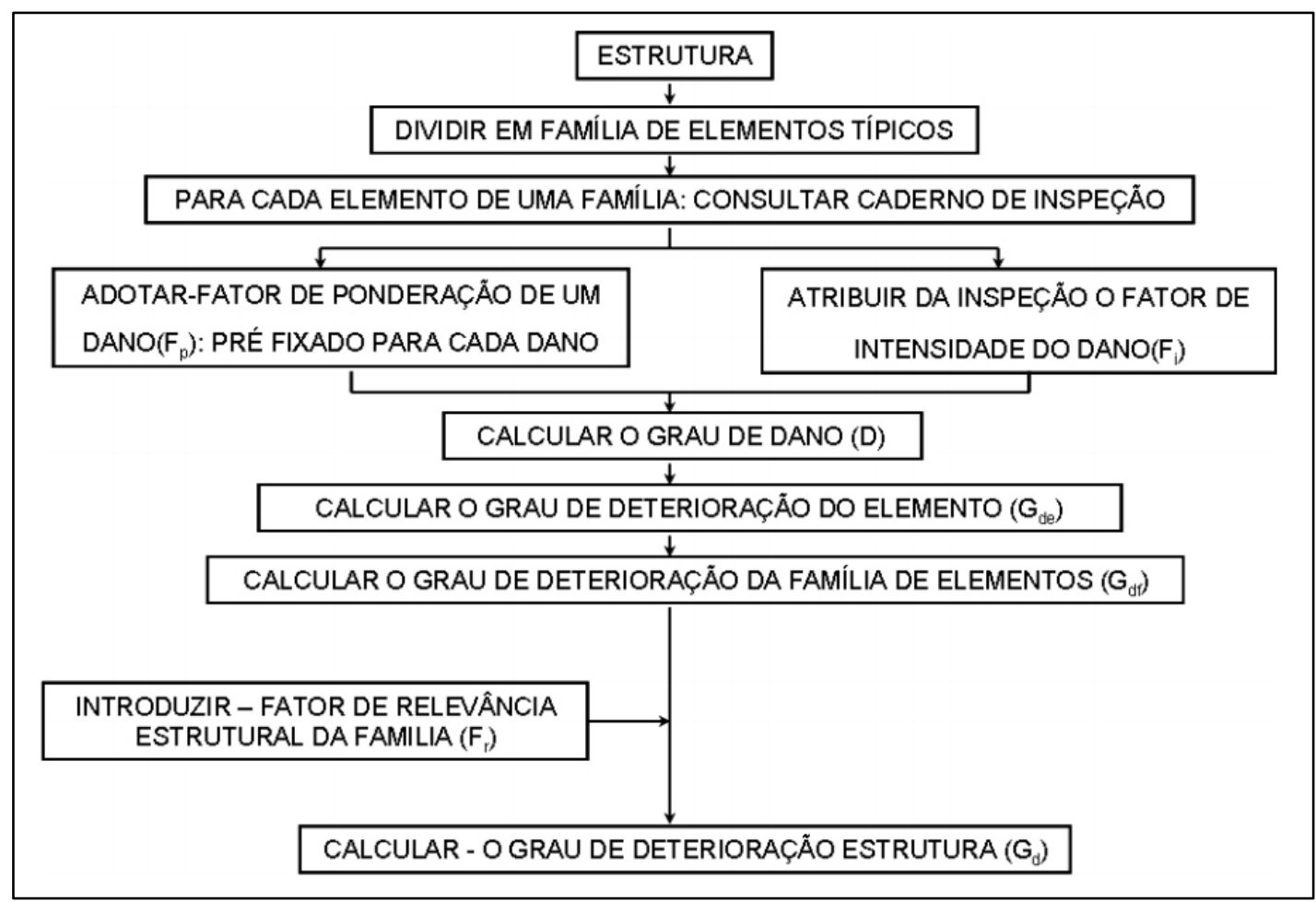

Figura 02. Fluxograma de utilização da metodologia GDE/UnB.

Fonte: Castro (1994) (adaptado).

Metodologia GDE/UNB aplicada na avaliação de uma edificação residencial 
A ideia é agrupar os elementos da edificação em famílias (ou grupos), sendo definida 6 (seis) tipos:

○ Pilares;

○ Vigas;

○ Vigas secundárias;

O Lajes;

- Elementos de composição arquitetônica; e

- Escadas e rampas.

2.2 Fator de ponderação $\left(F_{p}\right)$

O fator de ponderação varia de 01 a 05, sendo atribuído para valores dos danos causados pelas consequências patológicas, levando em conta a estética, funcionalidade e estabilidade dos elementos. Em outras palavras, esse parâmetro determina a importância relativa de uma determinada manifestação patológica, no que tange a segurança, funcionalidade e estética dos elementos de uma família. As tabelas 01 a 04 ilustram algumas dessas tabelas.

Tabela 01. Pilares

\begin{tabular}{lc}
\hline Danos & Fp \\
\hline Carbonatação & 3 \\
\hline Cobrimento Deficiente & 3 \\
Contaminação por cloretos & 4 \\
Corrosão de armaduras & 5 \\
Desagregação & 3 \\
Desplacamento & 3 \\
Descrio de geometria & 4 \\
Eflorescência & 2 \\
Falha de concretagem & 3 \\
Fissuras & 2 a 5 \\
Manchas & 3 \\
Recalque & 5 \\
Sinais de esmagamento & 5 \\
Umidade na base & 3 \\
\hline
\end{tabular}

Fonte: Fonseca, 2007.

Tabela 03. Lajes

\begin{tabular}{ll}
\hline Danos & Fp \\
\hline Carbonatação & 3 \\
Cobrimento Deficiente & 3 \\
Contaminação por cloretos & 4 \\
Corrosão de armaduras & 5 \\
Desagregação & 3 \\
Desplacamento & 3 \\
Eflorescência & 2 \\
Falha de concretagem & 2 \\
Fissuras & 2 a 5 \\
Flechas & 5 \\
Manchas & 3 \\
Sinais de esmagamento & 4 \\
Umidade & 3 \\
\hline
\end{tabular}

Fonte: Fonseca, 2007.
Tabela 02. Vigas

\begin{tabular}{ll}
\hline Danos & Fp \\
\hline Carbonatação & 3 \\
Cobrimento Deficiente & 3 \\
Contaminação por cloretos & 4 \\
Corrosão de armaduras & 5 \\
\hline Desagregação & 3 \\
Desplacamento & 3 \\
Eflorescência & 2 \\
Falha de concretagem & 2 \\
\hline Fissuras & 2 a 5 \\
\hline Flechas & 5 \\
\hline Manchas & 3 \\
\hline Umidade & 3 \\
\hline
\end{tabular}

Fonte: Fonseca, 2007.

Tabela 04. Escadas/Rampas

\begin{tabular}{ll}
\hline Danos & Fp \\
\hline Carbonatação & 3 \\
Cobrimento Deficiente & 3 \\
\hline Contaminação por cloretos & 4 \\
Corrosão de armaduras & 5 \\
Desagregação & 3 \\
Deslocamento por empuxo & 5 \\
Desplacamento & 3 \\
Desvio de geometria & 3 \\
\hline Eflorescência & 2 \\
Falha de concretagem & 2 \\
\hline Fissuras & 2 a 5 \\
Manchas & 3 \\
\hline Sinais de esmagamento & 5 \\
Umidade & 3 \\
\hline
\end{tabular}

Fonte: Fonseca, 2007. 


\subsection{Fator de intensidade $\left(\mathrm{F}_{\mathrm{i}}\right)$}

Esse fator relaciona a gravidade e evolução da manifestação patológica em um determinado elemento, variando entre 0 e 4 . Desta maneira:

- Elementos sem lesões $(\mathrm{Fi}=0)$;

- Elementos com lesões leves $(\mathrm{Fi}=1)$;

- Elementos com lesões toleráveis $(\mathrm{Fi}=2)$;

- Elementos com lesões graves $(\mathrm{Fi}=3)$;

- Elementos em estado crítico $(\mathrm{Fi}=4)$.

\subsection{Grau de dano (D)}

Em função do Fator de Ponderação do dano (Fp) e o Fator de Intensidade do dano (Fi), as equações (1) e (2) ilustram:

$$
\begin{array}{lll}
D=0,8 F i F p & \text { para } & F i \leq 2,0 \\
D=(12 F i-28) F p & \text { para } & F i>2,0
\end{array}
$$

2.5 Grau de deterioração de um elemento (Gde)

Esse indicador refere-se a um elemento individualmente, de acordo com a equação (3). A partir das informações obtidas para cada elemento, é possível determinar as ações necessárias.

$$
G d e=D m a ́ x\left[1+\frac{\sum_{i=1}^{n} D(i)-D m a ́ x}{\sum_{i=1}^{n} D(i)}\right]
$$

Onde:

Gde - Grau de deterioração de um elemento;

$D(i)$ - Grau de dano de ordem i;

$D_{\text {máx }}$ - Maior Grau de Dano;

$\mathrm{n}$ - número de danos encontrados em um elemento.

\subsection{Grau de deterioração de uma familia (Gdf)}

Esse parâmetro reune o conjunto de elementos que tenham as mesmas características, sendo calculado conforme a equação (4).

$$
G d f=G d e m a ́ x\left[\sqrt{1+\frac{\sum_{i=1}^{n} \operatorname{Gde}(i)-G d e m a ́ x}{\sum_{i=1}^{n} \operatorname{Gde}(i)}}\right]
$$

\subsection{Fator de relevância estrutural $\left(F_{r}\right)$}

O Fator de Relevância Estrutural $\left(\mathrm{F}_{\mathrm{r}}\right)$ deve ser aplicado para as famílias em conjunto, e do mesmo modo o fator de relevância estrutural da família de elementos, tendo o objetivo de considerar a importância relativa das diversas famílias que subdividem a estrutura, quanto ao comportamento e desempenho damesma. A tabela 05 sintetiza os valores.

Metodologia GDE/UNB aplicada na avaliação de uma edificação residencial 
Tabela 05. Fator de relevância estrutural $\left(F_{r}\right)$

\begin{tabular}{l|c}
\multicolumn{1}{c|}{ Familias em Conjunto } & $\begin{array}{c}\text { Fatores de Relevância } \\
\text { Estrutural }\left(\boldsymbol{F}_{r}\right)\end{array}$ \\
\hline Elementos de composição arquitetônica. & 1,0 \\
\hline Reservatório superior. & 2,0 \\
\hline $\begin{array}{l}\text { Escadas/rampas, reservatório inferior, cortinas, lajes secundárias, } \\
\text { juntas de dilatação. }\end{array}$ & 3,0 \\
\hline Lajes, fundações, vigas secundárias, pilares secundários. & 4,0 \\
\hline Vigas e pilares principais. & 5,0 \\
\hline
\end{tabular}

Fonte: Fonseca, 2007.

\subsection{Grau de deterioração global (Gd)}

Esse parâmetro é calculado a partir da média ponderada dos graus de deterioração das diversas famílias de elementos, com a implicação do Fator de Relevância Estrutural (Fr), como medida de majorar a importância de cada elemento ou famílias de elemento. A equação (5) ilustra o processo de cálculo.

$$
G d=\left[\frac{\sum_{i=1}^{n} \operatorname{Fr}(i) G d r(i)}{\sum_{i=1}^{n} \operatorname{Fr}(i)}\right]
$$

Para determinar as propostas de intervenções, com a finalidade de mitigar as patologias encontradas e recuperar a estrutura em estudo, utilizou-se o Grau de Deterioração do Elemento (Gde), de maneira isolada para facilitar.

O próximo item realça como foi realizado as propostas de intervenção e classificado a estrutura, ratificando o uso do grau de deterioração do elemento (Gde).

Salienta-se que embora haja o Grau de Deterioração de uma Família (Gdf) e o Grau de Deterioração Global, a pesquisa focou no Grau de Deterioração do Elemento (Gde).

\section{Materiais e métodos}

A figura 03 ilustra a metodologia definida para elaboração desse estudo, conforme os objetivos anteriormente.

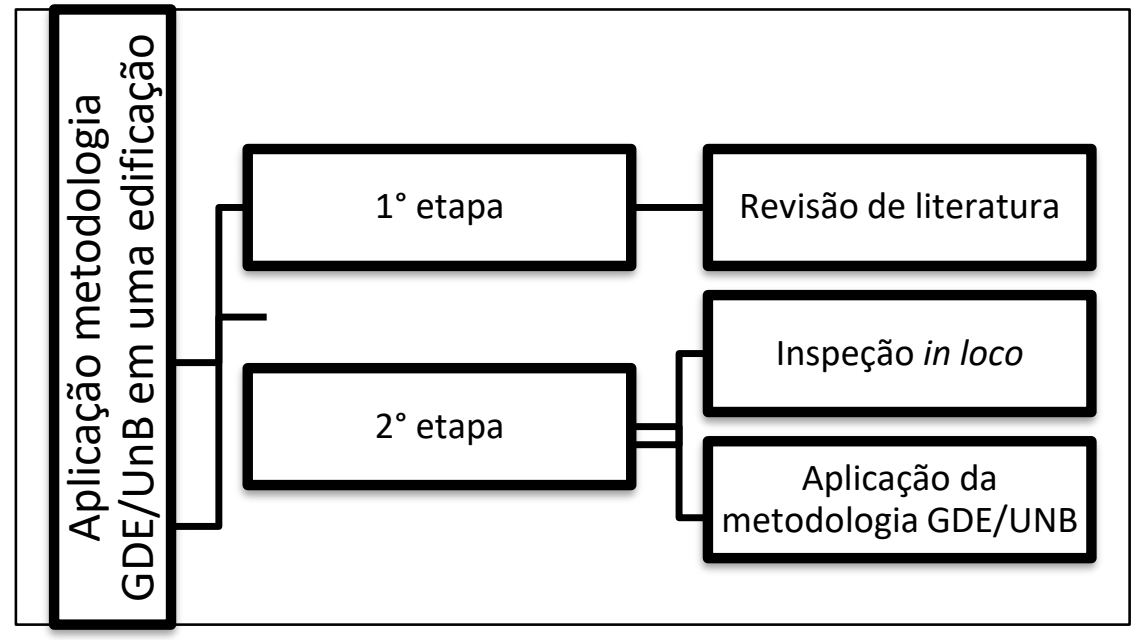

Figura 03. Metodologia considerada na execução do presente estudo. 
A partir do calculo da deterioração de um elemento (Gde), tem-se a classificação do nível de deterioração do elemento e ações recomendas, conforme metodologia GDE/UnB. A tabela pode ser encontrada em Fonseca (2007).

\section{Resultado e discussão}

Considerando a metodologia GDE/UnB descrita anteriormente, foram constatados altos níveis de deterioração em diversas anomalias encontradas na edificação em estudo, conforme pode ser verificado nas fotos 01 a 04.

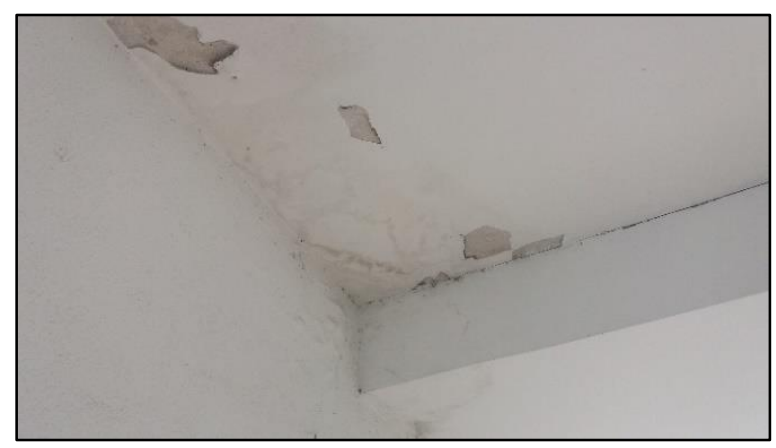

Foto 01. Manifestação patológica em laje.

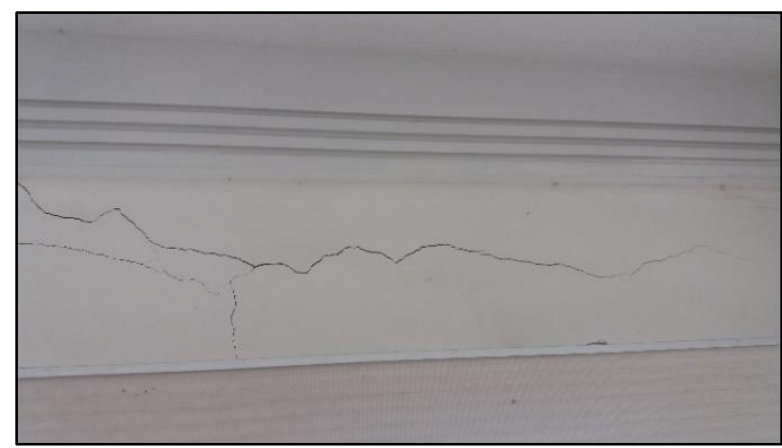

Foto 03. Manifestação patológica viga na sala.

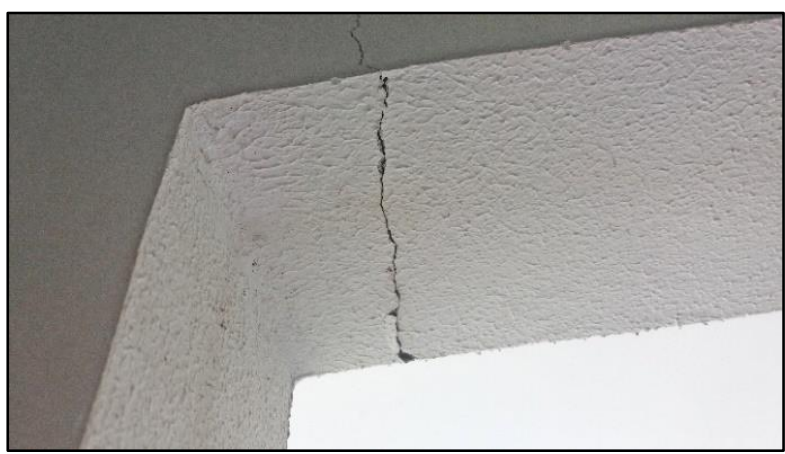

Foto 02. Manifestação patológica em viga.

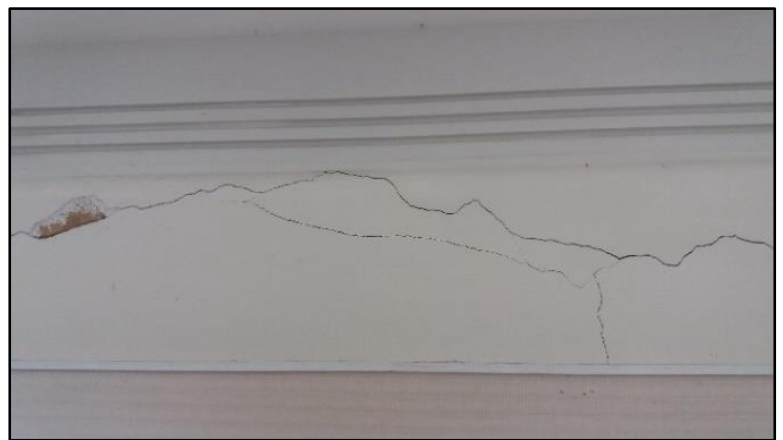

Foto 04. Manifestação patológica em viga na sala.

A tabela 06 sintetiza o Grau de Deterioração dos Principais elementos estruturais analisados, sabendo que não é possível expressar todos os resultados nesse artigo.

Tabela 06. Grau de Deterioração de Elemento (GDE) dos principais elementos encontrados.

\begin{tabular}{|c|c|c|c|c|c|}
\hline Nome do elemento & Dano (patologia) & Fp & Fi & D & \multirow{2}{*}{ Gde } \\
\hline \multirow{4}{*}{ Pilar mais crítico } & Fissuras & 4 & 3 & 32 & \multirow{3}{*}{26,67} \\
\cline { 2 - 5 } & Manchas & 3 & 3 & 24 & \\
\cline { 2 - 5 } & Corrosão de armaduras & 5 & 3 & 40 & \\
\cline { 2 - 5 } Viga mais crítica & Umidade base & 3 & 3 & 24 & \\
& Fissura & 4 & 3 & 32 & \multirow{3}{*}{26,67} \\
\cline { 2 - 5 } & Manchas & 3 & 3 & 24 & \\
\cline { 2 - 5 } & Corrosão de armaduras & 5 & 3 & 40 & \multirow{3}{*}{26,67} \\
\cline { 2 - 5 } & Umidade base & 3 & 3 & 24 & \\
\hline \multirow{3}{*}{ Laje (sala de estar) } & Fissura & 4 & 3 & 32 & \\
\cline { 2 - 5 } & Manchas & 3 & 3 & 24 & \\
\cline { 2 - 5 } & Corrosão de armaduras & 5 & 3 & 40 & \\
\cline { 2 - 5 } & Umidade base & 3 & 3 & 24 & \\
\hline
\end{tabular}

Metodologia GDE/UNB aplicada na avaliação de uma edificação residencial 
Como pode ser verificado, todos os principais elementos principais supracitados tiveram Gde equivalente a 26,67 (nível de deterioração médio), exigindo prazo de intervenção em até 2 (dois) anos. Vale ressaltar que grande parte dos elementos possui deterioração baixo a médio.

\section{Conclusão}

Diante dos resultados expostos, pode-se notar que a edificação apresenta-se em um estado médio de deterioração, principalmente devido a inexistência de manutenção preventiva, ocorrendo apenas o "mascaramento" das manifestações patológicas existentes (conforme indicado pelo proprietário). Nesse sentido, o desempenho global da estrutura encontra-se abaixo do mínimo exigido, necessitando de uma recuperação para restabelecer seu desempenho e aumentar sua vida útil.

Neste prisma, como os problemas patológicos são evolutivos e se agravam com o tempo, a interveção deve ser planejada e executada no menor tempo possível, com prazo máximo, conforme determinado, de 2 (dois) anos.

\section{Referências Bibliográficas}

BOLDO, P., Análise Quantitativa de Estruturas de Concreto Armado de Edificações no Âmbito do Exército Brasileiro. Dissertação de Mestrado, Publicação E.DM-001A/02, Departamento de Engenharia Civil e Ambiental, Universidade de Brasília, Brasília, DF, 2002, 295 p.

EUQUERES, P. Metodologia de inspeção em estruturas de pontes de concreto armado. Dissertação (Mestrado), Universidade Federal de Goiás, Goiânia, 2011. 168 p.

FONSECA, R. P. A estrutura do Instituto Central de Ciências: Aspectos históricos, científicos e tecnológicos de projeto, execução, intervenções e propostas de manutenção. Dissertação (Mestrado), Universidade de Brasília, Brasília, 2007. 213 p.

FONSECA, R. P. A estrutura do Instituto Central de Ciências: Aspectos históricos, científicos e tecnológicos de projeto, execução, intervenções e propostas de manutenção. Dissertação (Mestrado), Universidade de Brasília, Brasília, 2007. 213 p.

KLIMPEL, E.C. e SANTOS, P.R.C., Levantamento das manifestações patológicas presentes em unidades do conjunto habitacional Moradias Monteiro Lobato. Trabalho de Conclusão de Curso (Especialista em Patologia nas Obras Civis) - Instituto IDD, Curitiba, 2010, 98p.

MARTINS, M.S; HERNANDES, A. T.; MORIM, S. B. Ferramentas para melhoria do processo de execução dos sistemas hidráulicos prediais. In: III Simpósio Brasileiro de gestão e Economia da Construção, Anais, p.1619, set. 2003, São Carlos, 2003.

SOUZA, V.C.M e RIPPER, T., Patologia, recuperação e reforço de estruturas de concreto. Editora PINI, São Paulo, 1998.

VERLY, R. C., Comparação das metodologias de inspeção em obras de arte especiais: Gde/UnB e DNIT. Seminário de dissertação de mestrado, Departamento de Engenharia Civil e Ambiental, PECC, Universidade de Brasília, Brasília, DF, 2014. 